
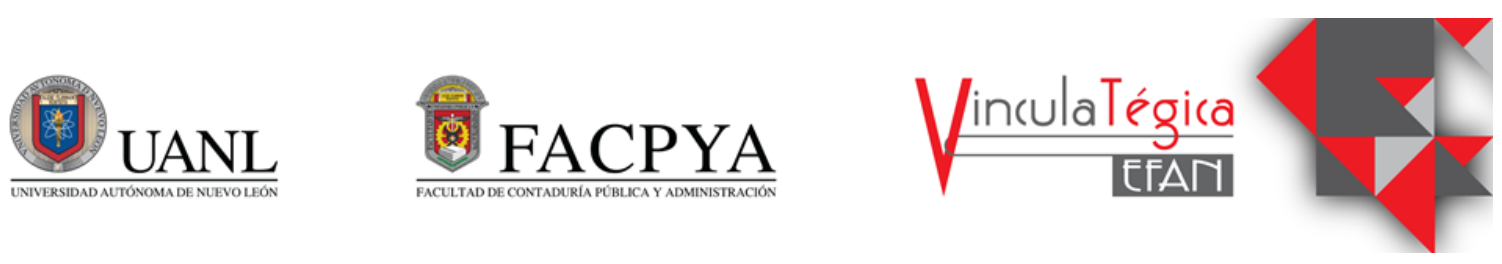

\title{
Desempeño del expositor y mejores prácticas como factores de satisfacción de los asistentes a webinars
}

\author{
Ángel Hernández Morales ${ }^{1}$, Santa Fe Martínez García ${ }^{2}$ y Omar Alonso Villafuerte \\ Sánchez ${ }^{3}$ \\ ${ }^{1}$ Universidad del Noreste, angel.hernandez@une.edu.mx, Av. Miguel Hidalgo 6315, Nuevo Aeropuerto, \\ 89337 Tampico, Tamps., 8332303830 ext 1122 \\ ${ }^{2}$ Universidad del Noreste, smartinez@une.edu.mx, Av. Miguel Hidalgo 6315, Nuevo Aeropuerto, 89337 \\ Tampico, Tamps., 8332303830 ext 1122 \\ ${ }^{3}$ Universidad del Noreste, ovillafuerte@une.edu.mx, Av. Miguel Hidalgo 6315, Nuevo Aeropuerto, 89337 \\ Tampico, Tamps., 8332303830 ext 1122
}

\begin{abstract}
Información del artículo revisado por pares
Fecha de aceptación: junio-2021

Fecha de publicación en línea: diciembre-2021

DOI: https://doi.org/10.29105/vtga7.1-160
\end{abstract}

\begin{abstract}
Resumen
Abstract

A raíz del distanciamiento social provocado por la As a result of the social distancing caused by COVID19, los eventos denominados como webinars han COVID19, events known as webinars have proliferado como una alternativa para distintas proliferated as an alternative for different organizaciones en varios sectores; ya que sus organizations in various sectors; since their características y beneficios que les confieren múltiples characteristics and benefits make them obtain ventajas en comparación a las conferencias presenciales. multiple advantages compared to face-to-face Sin embargo, el estudio sobre los comportamientos de la conferences. However, the study on audience audiencia en los webinars aún es incipiente. Por lo tanto, behaviors in webinars is still in its infancy. la finalidad de esta investigación fue la de identificar Therefore, the purpose of this research was to aquellos factores principales que inciden en la identify the main factors that affect the satisfaction satisfacción del asistente a los webinars. La presente of the webinar attendee. The present investigation investigación se caracterizó por ser cuantitativa, de was characterized by being quantitative, of alcance exploratorio descriptivo, no experimental. Para descriptive exploratory scope, not experimental. la recolección de los datos, se optó por recurrir a un To collect the data, it was decided to resort to a melf-administered survey, with questions on the encuesta autoadministrada, con reactivos en escala de Likert scale, dispersed online to the students of the Likert, dispersada vía online a los alumnos de la Universidad del Noreste (UNE), in the City and Universidad del Noreste (UNE), en la Ciudad y Puerto de Port of Tampico Tamaulipas. After obtaining 380 Tampico Tamaulipas. Después de obtenerse 380 validated observations, an Exploratory Factor observaciones validadas, se empleó un Análisis Factorial Analysis (EFA) with VARIMAX rotation was Exploratorio (AFE) con rotación VARIMAX. used.

En conclusión, el presente estudio encuentra que el Finally, this study finds that the exponent desempeño del expositor es un factor fundamental que performance is a fundamental factor that affects incide en la satisfacción del asistente al webinar, the satisfaction of the webinar attendee, destacando la efectividad para crear un espacio de highlighting the effectiveness to create a comfortable learning space and the enthusiasm
\end{abstract}


aprendizaje cómodo y el entusiasmo empleado durante la used during the webinar. Also, variables such as: exposición. Asimismo, las variables como: el diseño del course design, organization and technical aspects; curso, la organización y los aspectos técnicos; inciden en the la satisfacción del asiste al webinar y pueden ser considerados como un solo factor ligado a la planeación del webinar; ya que las acciones que dimensionan a estas variables tienen similitud teórica con las mejores prácticas para webinars.

Palabras clave: Webinar, satisfacción, desempeño del expositor, mejores prácticas. they affect the satisfaction of those attending the webinar and can be considered as a single factor linked to planning, since the actions that dimension these variables have theoretical similarity with the best practices for webinars.

Key words: Webinar, satisfaction, exponent performance, best practices

JEL Codes: I20, I29, O32, O33 y M31

\section{INTRODUCCIÓN}

A raíz del distanciamiento social provocado por la COVID19, la organización de webinars ha sido considerada como una alternativa para distintas organizaciones. Este argumento concuerda con el de Topor y Budson, quienes destacan que a raíz del confinamiento sanitario por la COVID19, los webinars "pueden ser la única opción para presentar información a un grupo de participantes" (2020, pág. 1216). El término o anglicismo denominado webinar, según Zoumenou $\mathrm{y}$ otros, hace referencia a "una presentación, seminario, conferencia o taller transmitido a través de Internet" (2015, pág. 62).

El webinar puede ser asíncrono o síncrono, siendo la segunda opción considerada la más avanzada, es una herramienta adoptada por diversas profesiones y se considera lo último en tecnología para el aprendizaje a distancia en vivo, debido a que las barreras para la participación son mínimas y se genera un nuevo nivel de conveniencia (Knipfer, y otros, 2019). Además, para otras organizaciones con fines distintos a los educativos, los webinars son un medio que les permite un acercamiento con posibles y actuales consumidores. El uso de webinars para entretenimiento o con fines comerciales es muy común en la actualidad (Oliver, 2020).

Los webinars tienen características distintivas y benéficas como la asequibilidad, la facilidad de uso y la capacidad informática para adaptarse a una gran demanda de asistentes, quienes pueden unirse a las sesiones en línea desde cualquier plataforma o dispositivo, siempre y cuando sean compatibles; asimismo, el acceso a estos eventos por lo regular es de modo gratuito dado que, en la mayoría de los casos, los costos operativos son asumidos por los organizadores o anfitriones del evento (Senecal \& Gazda, 2010). Estas características mencionadas, incluida la capacidad de interactuar con los participantes, hacen que este tipo de eventos tengan múltiples ventajas en comparación a las conferencias presenciales (Topor \& Budson, 2020); considerando que para asistir a un webinar los participantes solo requieren utilizar su computadora o dispositivo móvil (Zoumenou, y otros, 2015) y por obviedad, acceso a una red de Internet.

Otros beneficios que pueden ofrecer los webinars como recursos innovadores son: 1) la posibilidad de crecer en otros mercados; 2) ahorro y control del tiempo durante las presentaciones, rondas de preguntas y respuestas o interrupciones externas; 3) la digitalización de materiales, tanto del contenido como del resultado de la presentación; 4) carece de limitaciones geográficas; 5) se requiere menos logística; 6) la cantidad de asistentes puede ser mayor, en función de la capacidad de la plataforma seleccionada (Oliver, 2020). "El webinar también permite a los instructores archivar sus contenidos para que los alumnos accedan más adelante" (Alnabelsi, Al-Hussaini, \& Owens, 2015, pág. 270); no obstante, este último beneficio, hace que el webinar se vuelva asíncrono.

\subsection{Problema de investigación}

Para la presente investigación, resulta de interés analizar la naturaleza de los webinars, a razón de que se consideran como una herramienta tecnológica que coadyuva, de 
modo positivo, a mejorar el proceso de enseñanza/aprendizaje a través de una experiencia distinta. Actualmente, el aprendizaje en línea está mejorando el conocimiento de los actores del proceso educativo, el cual involucra a maestros, alumnos, personal universitario, técnicos y hasta los empresarios (Roff, 2018; Chopra, y otros, 2019). Además, el uso de webinars, se ha vuelto popular dentro de las estrategias del marketing educativo (Oliver, 2020). Asimismo, "proporcionan un complemento apropiado para el aprendizaje tradicional cara a cara, especialmente cuando existe la necesidad de flexibilidad de ubicación" (Ebner \& Gegenfurtner, 2019, pág. 9).

En contraste, "a pesar de su uso cada vez mayor y su conveniencia, los webinars siguen siendo una innovación reciente y las percepciones de los participantes no se han estudiado ampliamente" (Knipfer, y otros, 2019, pág. 842); y "a pesar de sus ventajas obvias, sorprendentemente se han realizado pocas investigaciones para evaluar el potencial de los eventos educativos síncronos en línea para brindar oportunidades de aprendizaje" (Alnabelsi, y otros, 2015, pág. 760). Estos argumentos concuerdan con la postura de Khajuria, quien expresa que "a pesar de la mayor utilización del seminario web como medio de enseñanza, hay estudios limitados" (2019, pág. 318); así como con la de Zoumenou, y otros (2015) quienes establecen que a pesar de que ya existía una tendencia por el uso de los webinars como recurso en las instituciones educativas; hay pocas investigaciones sobre las mejores prácticas necesarias para planificar y desarrollar estos eventos.

\subsection{Objetivo de la investigación}

Por lo tanto, la finalidad de esta investigación es identificar aquellos factores principales que inciden en la satisfacción del asistente a los webinars.

\subsection{Justificación}

Esta investigación se justifica con base en los tres criterios de importancia de los cinco mencionados por Hernandez Sampieri, Fernandez y Baptista (2010), los cuales son: la conveniencia, la relevancia social y la importancia práctica. Respecto a la conveniencia de la investigación, se establece que el presente estudio es un tema actual y que continuará generando atención, porque...

A medida que las tecnologías de aprendizaje electrónico avanzan rápidamente, existe una necesidad urgente de que los investigadores se mantengan al día con el estado actual de la tecnología en los contextos educativos para permitirles expandir el aprendizaje tradicional presencial mediante la introducción de modalidades de aprendizaje electrónico. (Ebner \& Gegenfurtner, 2019, pág. 9)

Respecto a la relevancia social, se alude a lo expuesto por Pedró (2020), quien señala que los webinars son un espacio o ventana de oportunidad para fomentar el aprendizaje móvil, debido a que para acceder a estos eventos online se puede disponer de dispositivos tecnológicos como tabletas electrónicas y teléfonos inteligentes, los cuales son de uso común entre los jóvenes quienes los suelen aprovechar para la continuidad educativa. De este modo, los webinars pueden ser utilizados por los asistentes como una herramienta para la preparación profesional y su desarrollo académico (Gegenfurtner, Zitt, \& Ebner, 2020). Aunado a esto, "la perspectiva futura es que este cambio tecnológico permitirá una educación de alta calidad a bajo costo" (Knipfer, y otros, 2019, pág. 841).

Respecto a la importancia práctica, se argumenta que las investigaciones "en el campo de las ciencias económicas y administrativas, en general son de carácter práctico, o bien, describen o analizan un problema o plantean estrategias que podrían solucionar problemas reales" (Bernal, 2010, pág. 106); por lo que, la presente investigación permitirá encontrar una serie de recomendaciones aplicables para la planeación y organización de webinars futuros producidas por las instituciones universitarias.

\section{MARCO TEÓRICO}

\subsection{La satisfacción en los webinars}

Sobre la satisfacción en los webinars o en ambientes catalogados como aprendizaje en línea, la presente investigación alude a varios 
estudios como el de Mason y Weller (2000), quienes proponen tres factores que afectan la satisfacción de los estudiantes en cursos en línea, los cuales son: 1) el apoyo de su tutor u otro personal o estudiantes, 2) la cantidad de tiempo, paciencia y motivación que los alumnos deben dedicar al curso; y 3) que el contenido y la presentación del curso se ajustan a las expectativas y al estilo de aprendizaje de los estudiantes.

Por su parte, Bekele (2010), en su estudio se propone por objetivo examinar los impactos generales de entornos de aprendizaje apoyados por Internet sobre la satisfacción y motivación en la educación superior, indicó que "los atributos de la tecnología, la calidad del curso, el compromiso, el formato del programa y los servicios de apoyo respaldan la motivación y la satisfacción" (pág. 116). Para Alqahtani y Mohammad (2015) la satisfacción del alumno, en aprendizajes mediante dispositivos electrónicos conectados a Internet, puede verse influida por la utilidad percibida del sistema y la calidad del contenido.

En la investigación realizada por Kukreja, Kaur y Aggarwal (2021), identifican los factores que inciden en la satisfacción del estudiante con las clases en línea durante la pandemia derivada por la COVID-19, estos son: la calidad del instructor, el diseño del curso, la orientación hacia las tecnologías de la información y comunicación (TIC's), así como la conciencia, la mentalidad abierta y la amabilidad por parte de los asistentes; todos estos factores muestran, en los resultados del estudio, una relación positiva en la satisfacción de los estudiantes.

Por lo tanto, derivado de la revisión teórica sobre la satisfacción en los webinars y en ambientes de aprendizaje en línea, la presente investigación propone un conglomerado de cuatro factores incidentes en la satisfacción de los asistentes a los webinars, los cuales son: 1) el desempeño del expositor, 2) el diseño del contenido, 3) la organización del webinar y 4) los aspectos tecnológicos. Cabe mencionar que, en el presente estudio, se consideran solo aquellos factores exclusivos y del alcance de los organizadores o proveedores del webinar. Por lo que, para beneficio y alcance del objetivo de la investigación, se excluyen aquellos factores relativos al desempeño, disposición, perfil o cualquier otro rol que dependa del asistente, debido a que son factores o aspectos que carecen del control parcial o total por parte del organizador.

\subsubsection{El desempeño del expositor}

El desempeño del facilitador o expositor es el primer factor propuesto en esta investigación, debido a que es una variable continuamente mencionada a través de la revisión teórica. $\mathrm{La}$ eficiencia docente es una variable crítica para la satisfacción de los estudiantes (Munteanu, y otros, 2010; Kukreja, y ot ros, 2021). Los asistentes a espacios de aprendizaje en línea o webinars creen en la importancia de que un profesor, facilitador o expositor esté capacitado e informado sobre los materiales del curso, así como proporcionar estrategias efectivas y con conocimientos pedagógicos actualizados (Lee, 2014).

Además, cuando los alumnos tienen una mayor interacción con el formador se puede lograr aumentar los niveles de satisfacción y, en última instancia, contribuir a niveles más altos de aprendizaje (Gegenfurtner, Zitt, \& Ebner, 2020). Finalmente, la revisión de la literatura revela que "el instructor es el factor más importante que afecta a la satisfacción del estudiante" (Kukreja, Kaur, \& Aggarwal, 2021, pág. 370). Por lo tanto, se puede establecer la siguiente hipótesis:

H1: El desempeño del expositor del webinar incide en la satisfacción del asistente.

\subsubsection{El diseño del contenido}

En primera instancia, se considera que este factor tiene una influencia en el nivel de aprendizaje y posteriormente incide en la satisfacción. Knipfer, y otros (2019), se trazan como objetivo de este estudio evaluar la aceptación de los estudiantes de un seminario web para la educación médica continua, de esta manera resuelven que el aprendizaje de los asistentes es significativo y se refiere a la percepción sobre el contenido y su relevancia práctica para el estudiante.

Asimismo, Kukreja, y otros, (2021) encuentran que "el diseño del curso tiene un impacto positivo $y$ significativo en la 
satisfacción de los estudiantes con las clases en línea" (pág., 370). Lee (2014) se refiere a que el diseño del curso debe proporcionar una estructura clara de contenidos, pautas y rúbricas para que el aprendizaje en línea sea satisfactorio. Por lo tanto, se puede establecer la siguiente hipótesis:

$\mathrm{H} 2$ : El diseño del contenido del webinar incide en la satisfacción del asistente.

\subsubsection{La organización del webinar}

La organización adecuada del curso tiene un impacto positivo en la satisfacción de los participantes si se logra aumentar los niveles de participación, fomentar diseños de instrucción centrados en el alumno con herramientas como encuestas $y$ foros de discusión, minimizar el tiempo necesario para discutir las soluciones de tareas de manera colaborativa, ofrecer grabaciones de la sesión del webinar, aclarar preguntas y abordar cualquier problema o laguna de conocimiento con contenidos poco complejos o difíciles para profundizar en los que ya se han cubierto en sesiones presenciales y considerar el tiempo de duración, que por lo regular en los webinars, no debieran exceder los 90 minutos (Gegenfurtner, Zitt, \& Ebner, 2020). Por lo tanto, se puede establecer la siguiente hipótesis:

H3: La organización del webinar incide en la satisfacción del asistente.

\subsubsection{Aspectos técnicos}

Los aspectos técnicos juegan un rol importante en el modo en que los asistentes experimentan los webinars y al mismo tiempo, contienen un grado de complejidad en el desarrollo del evento; ya que, en algunas ocasiones, los participantes suelen informar de problemas técnicos para acceder al seminario web (Gschwandtner, 2016) o cualquier otra vicisitud durante el webinar. Por lo que, los organizadores deben de prevenir, hasta donde sea posible, aquellas dificultades que puede ocasionar la variable tecnológica.

Los asistentes al webinar requieren de un sistema o plataforma fácil de usar para lograr su satisfacción, ya que algunos estudiantes carecen de un elevado dominio del uso de la tecnología; de ahí que es importante proporcionar un sistema fácil, amigable e intuitivo y si es necesario, proporcionar una inducción sobre cómo disponer de los materiales del curso, cómo obtener asistencia técnica, cómo acceder al evento, etc., para reducir el nivel de frustración de los estudiantes y evitar que las dificultades con la tecnología en línea interfieran con el aprendizaje e incidan negativamente en la satisfacción (Lee, 2014). De acuerdo a lo anterior, se puede establecer la siguiente hipótesis:

H4: Los aspectos técnicos del webinar inciden en la satisfacción del asistente.

\subsection{Modelo gráfico de la hipótesis} Como se observa en la Figura 1, en el modelo gráfico de la hipótesis se observan las variables independientes $\mathrm{X} 1, \mathrm{X} 2, \mathrm{X} 3 \mathrm{y}$ $\mathrm{X} 4$, y como inciden en la dependiente $\mathrm{Y} 1$.

Figura 1. Factores incidentes en la satisfacción del asistente y compatibles con las buenas prácticas.

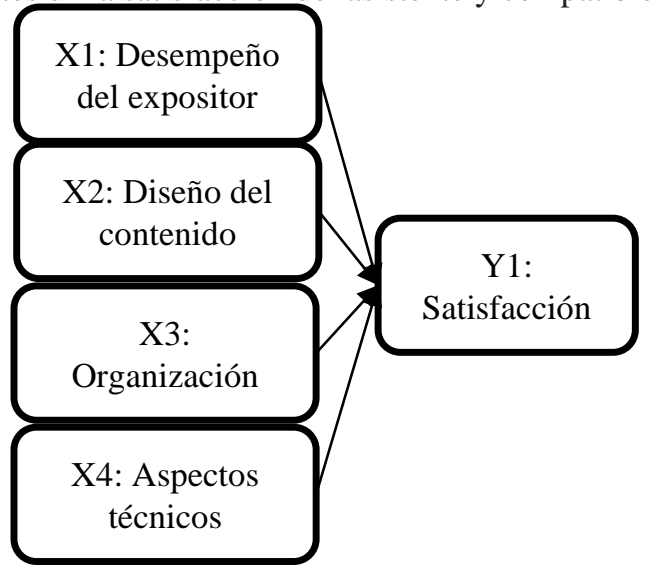

Fuente: Elaboración propia 


\section{MÉTODO}

La presente investigación se caracteriza por ser cuantitativa, de alcance exploratorio descriptivo, no experimental. Para la recolección de los datos, se optó por recurrir a una encuesta autoadministrada, la cual fue una adaptación a partir de ítems empleados por tres instrumentos distintos (Lee, 2014; Khajuria, 2019; Knipfer, y otros, 2019). Los ítems fueron medidos con escala de Likert de 10 niveles y se utilizó un Análisis Factorial Exploratorio (AFE) con rotación VARIMAX, con la finalidad de extraer los factores de mayor carga significativa. La encuesta fue dispersada de modo online, por medio de la plataforma Google Forms, mediante la liga electrónica

https://forms.gle/gJHY2KZH7sQJmRD67.

$\mathrm{El}$ instrumento fue dividido en cinco secciones. La primera sección fue con reactivos de filtro y su finalidad era la de asegurar que el encuestado cumpliese con los requerimientos demográficos, mencionados posteriormente en el epígrafe 3.2; y de esta manera validar las observaciones obtenidas. Las siguientes cuatro secciones fueron empleadas para medir la incidencia de cada variable sobre la satisfacción. Para cada sección, se empleó un enunciado declarativo sobre la satisfacción como asistente al webinar y se solicitó al encuestado seleccionar su percepción en una escala de Likert de 10 niveles, donde el nivel mínimo era completamente en desacuerdo y el nivel máximo era totalmente de acuerdo.

Para la X1, desempeño del expositor, los ítems fueron: P1) comunicación efectiva, P2) entusiasmo del expositor, P3) preocupación por el aprendizaje, P4) respeto por el aprendizaje de los asistentes, P5) accesibilidad para la atención y el seguimiento de los interesados, P6) efectividad para crear un espacio de aprendizaje cómodo y P7) efectividad para promover las interacciones. Para la X2, diseño del contenido, los ítems fueron: P8) curso intelectualmente desafiante y estimulante, P9) aprendizaje valioso, 10) aumento del interés por el tema y 11) comprensión por los materiales del curso. Para la X3, organización del curso, los ítems fueron: P12) explicaciones del instructor claras, P13) preparación de los materiales del curso, P14) compatibilidad entre el objetivo y el desarrollo del curso y P15) temas fáciles de resumir en notas escolares. Por último, para la variable $\mathrm{X} 4$, aspectos técnicos, los ítems utilizados fueron: P16) distribución de los materiales en línea, P17) utilización de elementos multimedia, P18) utilización de un panel de discusión y P19) apoyo o asistencia técnica.

\subsection{Estimación de la muestra}

Para la estimación de la muestra se consideran tres aspectos característicos de la presente investigación. Primero, aunque se conoce el número actual de estudiantes de la Universidad del Noreste (UNE), que en un principio podría suponer que el universo de la investigación es finito, se desconoce con exactitud si los estudiantes de dicha institución fueron en realidad asistentes con permanencia total y síncrona durante alguno de los diferentes webinars que se realizaron de modo particular desde el mes de mayo del 2020 a enero 2021; ya que el control de la asistencia y permanencia en los eventos en línea habían sido poco certeros en un inicio, sobre todo en aquellas plataformas utilizadas que carecen de herramienta de registro de asistencia y control de permanencia. Segundo, el empleo de un AFE para el manejo y análisis estadístico de los datos. Por último, el uso de reactivos medidos con escala de Likert de 10 niveles.

En consecuencia, debido a estas características de la presente investigación, se hace referencia a la propuesta estadística planteada por Rositas (2014), la cual argumenta que con las características mencionadas de la investigación...

La media tiende a 5.5 entonces, la varianza tiende a 7 . Tomando esto como referencia, y con un $95 \%$ de confianza y un error tolerable de $\mathrm{d} \pm 0.275$ (el $5 \%$ de 5.5) que utilizaríamos como valor de la precisión "d" cuando se haga la estimación por intervalo del parámetro poblacional... (pág. 252)

Por lo tanto, la estimación total de la muestra de esta investigación es de 368 
observaciones mínimo. La fórmula desarrollada fue la siguiente:

Figura 2. Estimación de la muestra.

$$
n=\frac{Z_{a} / 2 * s^{2}}{d^{2}} n=\frac{1.96^{2} * 7^{2}}{(1-.275)^{2}}
$$

Fuente: Elaboración partir de Rositas Rositas, J. (Los tamaños de las muestras en encuestas de las ciencias sociales y su repercusión en la generación del conocimiento, 2014)

\subsection{Delimitación del estudio}

Respecto a cuestiones demográficas, los participantes requeridos para contestar la encuesta fueron estudiantes universitarios, entre los 17 y 28 años, quienes expresan haber participado como asistentes a un webinar desde el mes de mayo del 2020 a enero 2021. De acuerdo con la delimitación espacial, se comenta que el estudio se realizó en el Área de Negocios y Humanidades (ANH) de la UNE, en la ciudad y puerto de Tampico Tamaulipas, México. Por último, debido a que la recolección de datos fue durante el primer trimestre del año 2021, la presente investigación es de corte transeccional.

\section{RESULTADOS}

De modo inicial, se realizó una prueba piloto con 29 observaciones, en donde se obtuvo un análisis de fiabilidad con un Alpha de Cronbach de .951, un coeficiente de KMO de .652 y una esfericidad de Barlett de .000; lo que hizo suponer que el instrumento era estadísticamente fiable y que con más observaciones la prueba de KMO mejoraría. Asimismo, en la misma prueba piloto se observó que en el AFE se alcanzaba un $83.275 \%$ de varianza explicada con cuatro componentes, lo que hizo suponer que el modelo estadístico era compatible con el modelo hipotético.

Finalmente, después de las mejoras realizadas al instrumento a raíz de el pilotaje y la revisión de expertos del Comité de Investigación del ANH de la UNE, la encuesta se dispersó y se obtuvieron 401 observaciones totales, de las cuales se consideraron solo 380 como válidas, superando lo propuesto en la estimación de la muestra. Con esta cantidad de observaciones, el AFE obtuvo un coeficiente de KMO de .970 y una esfericidad de Barlett de .000, lo cual indicaba que la prueba estadística seleccionada era ideal para el análisis de los datos. Sin embargo, la varianza total explicada, la cual fue $76.213 \%$ y que resultó estadísticamente significativa, se alcanzó con sólo dos componentes, ambos con una carga factorial de .753 .

Este resultado genera una disyuntiva. Por un lado, el obtener como resultado solo dos componentes es poco favorable para la investigación, ya que se contrapone al modelo hipotético el cual planteaba cuatro factores. Por el otro lado, la varianza explicada y las cargas factoriales de los componentes del modelo estadístico son significativos. Además, la variable X1 desempeño del expositor, logró conglomerar todos los ítems propuestos con cargas significativas y logra una carga factorial de .753 , tal y como se muestra en la columna denominada como F2 de la Tabla 1.

Consecuentemente, derivado de la rotación de los componentes del AFE, se observa que de los 19 ítems que dimensionan los factores del modelo propuesto, sólo dos de ellos carecen de una carga factorial estadísticamente significante; estos son: el P18 con .656 y el P8 con 6.29. El resto supera la carga factorial mínima propuesta para este estudio, la cual es de .700. Asimismo, los ítems P16, P12, P17 y P6, presentan cargas superiores al resto. Tal y como se puede apreciar en la Tabla 2, donde la columna denominada como F1 y F2, hacen alusión a los componentes donde los ítems fueron conglomerados.

Tabla 1. Matriz de transformación de componentes. 


\begin{tabular}{lll}
\hline Componente & F1 & F2 \\
\hline F1 & .753 & .658 \\
F2 & -.658 & .753 \\
\hline
\end{tabular}

Fuente: Elaboración propia

Tabla 2. Matriz de transformación de componentes.

\begin{tabular}{lll}
\hline Ítem & F1 & F2 \\
\hline P16 Distribución de los materiales en línea & $\mathbf{. 8 3 4}$ & .365 \\
P12 Las explicaciones del instructor son claras & $\mathbf{. 8 3 0}$ & .352 \\
P17 La utilización de elementos multimedia & $\mathbf{. 0 8}$ & .338 \\
P15 El instructor desarrolla charlas que facilitan la toma de & $\mathbf{. 7 9 7}$ & .428 \\
notas & & \\
P11 Comprensión de los materiales del curso & $\mathbf{. 7 9 3}$ & .436 \\
P14 Los objetivos coinciden con la finalidad del curso & $\mathbf{. 7 7 0}$ & .469 \\
P13 Los materiales del curso están bien preparados y & $\mathbf{. 7 5 4}$ & .474 \\
cuidadosamente explicados & & \\
P10 Aumento del interés por el tema & $\mathbf{. 7 3 9}$ & .414 \\
P19 El apoyo o asistencia técnica & $\mathbf{. 7 2 9}$ & .451 \\
P9 Aprendizaje valioso & $\mathbf{. 7 0 6}$ & .520 \\
P18 El uso de un panel de discusión & $.656^{* *}$ & .468 \\
P8 Curso intelectualmente desafiante y estimulante & $.629 * *$ & .503 \\
P6 Efectividad para crear un espacio de aprendizaje cómodo & .421 & $\mathbf{. 8 0 8}$ \\
P2 Entusiasmo durante la exposición en línea & .368 & $\mathbf{. 7 9 0}$ \\
P3 Preocupación por el aprendizaje de los asistentes & .364 & $\mathbf{. 7 8 3}$ \\
P1 Una comunicación efectiva & .385 & $\mathbf{. 7 7 6}$ \\
P7 Efectividad para promover las interacciones & .431 & $\mathbf{. 7 5 2}$ \\
P4 Respeto por el aprendizaje de los asistentes & .431 & $\mathbf{. 7 4 9}$ \\
P5 Accesibilidad para la atención y el seguimiento fuera del & .480 & $\mathbf{. 7 0 6}$ \\
curso & &
\end{tabular}

Fuente: Elaboración propia

Nota: ** ítems con cargas poco significativas

\section{CONCLUSIONES RECOMENDACIONES}

La presente investigación se ha focalizado en el estudio de los webinars. A raíz del distanciamiento social provocado por la COVID19, estos eventos han proliferado como una alternativa para distintas organizaciones en varios sectores. Las características y beneficios de los webinars hacen que estos obtengan múltiples ventajas en comparación a las conferencias presenciales. Sin embargo, el estudio sobre los comportamientos de la audiencia en los webinars aún es incipiente. Por lo tanto, la finalidad de esta investigación fue la de identificar aquellos factores principales que inciden en la satisfacción del asistente a los webinars. Por tal razón, después de la revisión teórica se propusieron hipotéticamente cuatro factores que inciden en la satisfacción de un asistente: X1 el desempeño del expositor, X2 el diseño del contenido, X3 la organización del webinar y $\mathrm{X} 4$ los aspectos tecnológicos.

Después del AFE, aplicado a los datos recolectados, se puede concluir que la variable $\mathrm{X} 1$ desempeño del expositor, es sin lugar a duda, el factor principal que incide en la 
satisfacción de los asistentes. Este hallazgo concuerda con estudios empíricos previos (Munteanu, y otros, 2010; Lee, 2014; Kukreja, y otros, 2021). Siendo la efectividad para crear un espacio de aprendizaje cómodo, P6, la dimensión más importante de este factor. Sin embargo, el modelo hipotético tiene severas consideraciones. Porque, aunque la mayoría de los ítems logran una carga factorial significativa, las variables $\mathrm{X} 2$ el diseño del contenido, X3 la organización del webinar y $\mathrm{X} 4$ los aspectos tecnológicos, se fusionan y son conglomeradas en un solo factor denominado F1.

Este resultado no se observó durante el piloteo. No obstante, se pudo haber previsto por el resultado elevado del coeficiente del Alpha de Cronbach, el cual era de .951 durante el piloteo; porque, en ocasiones, cuando este resultado es mayor de 0.90, "debemos asegurarnos que algunos de los ítems, no sean sino mero parafraseos de otros ítems" (Rositas, 2014, pág. 259). Por ende, se generan los siguientes supuestos que limitaron el estudio y que pudieron ocasionar la fusión de estas variables en un solo factor.

Primero, la adaptación del instrumento pudo tener un sesgo metodológico a pesar de las pruebas de confiabilidad tanto de expertos como de las pruebas estadísticas. Segundo, si bien la investigación considera variables con sustento teórico para la construcción del modelo hipotético y del instrumento, se optó por excluir aquellas variables que eran consideradas como aspectos relativos al comportamiento o desempeño del asistente, como la disposición, el perfil o el motivo de participación del asistente; debido a que el enfoque de esta investigación era para profundizar en la satisfacción del asistente desde la perspectiva del organizador o proveedor, decisión que pudo influir en el resultado del análisis estadístico. Tercero y último, se puede generar el supuesto de que la naturaleza de las variables $\mathrm{X} 2$, $\mathrm{X} 3$ y X4, tienen rasgos y características semejantes, con una estrecha relación de aspectos orientados a la etapa de la planeación de los webinars.

La explicación del último supuesto radica en que para lograr la satisfacción de los asistentes al webinar, es necesario cumplir con ciertas acciones que son comprendidas desde la planificación de los aspectos técnicos, las actividades diseñadas para el cumplimiento del objetivo pedagógico del curso, la organización para la interacción de los asistentes durante y después del evento. Estas acciones planificadoras ya han sido estudiadas y se les conoce como mejores prácticas. Zoumenou, y otros, (2015), establecen como concepto de mejores prácticas al conjunto de las acciones ocurridas "antes, durante y después del webinar, de modo que los participantes se sientan atraídos a aprender, permanecer comprometidos y beneficiarse intelectualmente de esta innovación pedagógica" (pág. 62).

Senecal y Gazda (2010) plantean algunas estrategias, consideradas también como mejores prácticas de un webinar y que inician desde la planeación; ya que, para estos autores, "el éxito inicial incluye una planificación adecuada, la voluntad de adaptarse según sea necesario y la prevención de tantos problemas con anticipación como sea posible" (pág. 15). Las estrategias expuestas por los autores mencionados son: diseñar estrategias claras, detallar requisitos técnicos, evaluar actividades adecuadas, instruir al expositor sobre la plataforma, diseñar información gráfica sobre el webinar, designar roles de apoyo y asistencia técnica, instruir a los asistentes sobre el desarrollo, exhortar sobre el tipo de conectividad y dispositivo adecuados y seleccionar funciones interactivas.

Topor y Budson (2020) proponen algunos aspectos claves para el éxito de un webinar como la de planificar la logística del evento, la evaluación de las necesidades dependiendo la naturaleza del webinar, desarrollar objetivos específicos de aprendizaje, empatizar sobre las necesidades de los asistentes, material de presentación claro y atractivo, desarrollar actividades de interacción, familiarizarse con la plataforma seleccionada y tener un plan alternativo en caso de presentarse fallas técnicas, realizar prácticas del evento, organizarse y prepararse con los recursos disponibles, evaluar el aprendizaje de los participantes, utilizar los comentarios y observaciones de la audiencia 
para mejorar el desempeño del evento y finalmente, que tanto el organizador como el expositor compartan experiencias para una retroalimentación previa y posterior al webinar

De esta manera y haciendo énfasis sobre el alcance metodológico de este documento, el cual es exploratorio descriptivo, resulta conveniente continuar con esta línea de investigación sobre la satisfacción en los webinars, con las siguientes recomendaciones. Primero, construir el contexto necesario y óptimo, en medida de lo posible y en base a las acciones de planificación consideradas como mejores prácticas, para realizar un estudio cuasiexperimental que indague con mayor profundidad la satisfacción de los asistentes a un webinar a partir de los hallazgos producidos en este documento; dichas acciones se pueden apreciar en la Tabla 3. Segundo, adicionar a los futuros estudios variables ligadas al rol del asistente como la motivación o el interés hacia el webinar, el estado de ánimo del asistente y la compatibilidad entre el tema del webinar con el perfil del asistente; con la finalidad de mejorar el modelo. Tercero, construir instrumentos de medición validados por métodos cualitativos y cuantitativos. Por último, robustecer el análisis de datos con pruebas estadísticas de mayor complejidad como las Ecuaciones Estructurales Múltiples (MES).
Finalmente y en conclusión, el presente estudio encuentra que el desempeño del expositor es un factor fundamental que incide en la satisfacción del asistente al webinar, destacando la efectividad para crear un espacio de aprendizaje cómodo y el entusiasmo empleado durante la exposición; además debe considerarse la comunicación efectiva, la preocupación por el aprendizaje, el respeto por el aprendizaje de los asistentes, la accesibilidad para la atención de los interesados y la efectividad para promover las interacciones. Por lo que, la $\mathrm{H} 1$ es aceptada.

Asimismo, la organización, el diseño del curso y los aspectos técnicos inciden en la satisfacción del asistente al webinar; empero, pueden ser consideradas como una sola variable ligada a la planeación, ya que las acciones que dimensionan a estas variables tienen similitud con las mejores prácticas para los webinars. Además, el crear un panel de discusión y la percepción del nivel de lo desafiante y estimulante que puede ser el webinar son aspectos que no logran incidir en la satisfacción de modo estadísticamente significativo. Por lo que, habría que valorar si en realidad los paneles de discusión y el nivel de desafío del webinar inciden en la satisfacción del asistente o habría que mejorar o reformular el ítem con el que fueron analizados. Por lo que, la H2, H3 e H4, son aceptadas, pero con reservas.

Tabla 3 Estrategias de planeación para las buenas prácticas de un webinar.

\begin{tabular}{|c|c|c|}
\hline Acción & Desarrollo & Finalidad \\
\hline $\begin{array}{c}\text { Diseñar } \\
\text { estrategias claras. }\end{array}$ & $\begin{array}{c}\text { El instructor organiza el curso de manera que las } \\
\text { experiencias de aprendizaje sincrónico sean consistentes } \\
\text { y estructuradas para el contexto. }\end{array}$ & $\begin{array}{l}\text { Aumentar la interacción } \\
\text { y el nivel de } \\
\text { participación. }\end{array}$ \\
\hline $\begin{array}{l}\text { Detallar los } \\
\text { requisitos } \\
\text { técnicos. }\end{array}$ & $\begin{array}{c}\text { Proporcionar recomendaciones técnicas, pero } \\
\text { fundamentadas en cuestiones pedagógicas, que logren } \\
\text { coadyuvar al proceso de enseñanza/aprendizaje o al } \\
\text { objetivo específico del webinar. }\end{array}$ & $\begin{array}{l}\text { Evitar problemas, que } \\
\text { puedan frustrar a todos } \\
\text { los participantes. }\end{array}$ \\
\hline $\begin{array}{l}\text { Evaluar las } \\
\text { actividades } \\
\text { adecuadas. }\end{array}$ & $\begin{array}{c}\text { El instructor u organizador deben rediseñar o } \\
\text { proporcionar un contexto alternativo para aquellas } \\
\text { actividades que carecen de una naturaleza síncrona, } \\
\text { como la elaboración de un producto, la resolución de } \\
\text { cuestionario o el reporte o análisis de una lectura, por } \\
\text { mencionar algunas. }\end{array}$ & $\begin{array}{l}\text { Aumentar la interacción } \\
\text { y el nivel de } \\
\text { participación. }\end{array}$ \\
\hline $\begin{array}{l}\text { Capacitar al } \\
\text { instructor sobre la } \\
\text { plataforma. }\end{array}$ & $\begin{array}{l}\text { El organizador debe orientar al instructor mediante } \\
\text { recomendaciones de la plataforma, su uso y } \\
\text { herramientas; así como una introducción sobre las }\end{array}$ & $\begin{array}{l}\text { Evitar problemas, que } \\
\text { puedan frustrar a todos } \\
\text { los participantes. }\end{array}$ \\
\hline
\end{tabular}


actividades de aprendizaje sincrónico que pueden ejecutarse.

\author{
Diseñar \\ información \\ gráfica sobre el \\ webinar. \\ Designar roles de \\ apoyo.
}

Instruir a los asistentes sobre el desarrollo del webinar.

Exhortar sobre el uso de dispositivos y el tipo conectividad.

Seleccionar funciones interactivas.
El organizador debe generar diagramas de flujo o instructivos gráficos para el acceso fácil y casi intuitivo de la transmisión.

El organizador debe proveer de un "sistema de acompañamiento" durante el webinar nombrando a un moderador y en caso de ser requerido, un asistente para aspectos técnicos.

El organizador debe de propiciar algún tipo de orientación o en caso de ser requerido práctica previa, para aquellos usuarios que no están familiarizados con la plataforma.

El organizador puede hacer referencia sobre el tipo de dispositivos, las características o hardware necesarios, la calidad del equipo y la capacidad mínima de conectividad a internet necesarios para el webinar.

Tanto el organizador como el instructor pueden, en un momento dado durante el webinar, seleccionar algunas funciones interactivas de la plataforma $u$ otras que puedan adaptarse al momento. De preferencia dos o máximo tres para evitar que los asistentes se sientan abrumados y sobre estimulados.
Evitar confusiones en el punto de acceso.

Ayudar y mantener a los participantes organizados.

Reducir problemas durante el desarrollo del webinar.

Reducir problemas durante el desarrollo del webinar.

Aumentar la interacción y el nivel de participación.

Fuente: Elaboración a partir de Senecal y Gazda (Harmonizing the Virtual Choir: Interactive Synchronous Webinars for Online Education, 2010, pág. 15) 


\section{REFERENCIAS}

Alnabelsi, T., Al-Hussaini, A., \& Owens, D. (2015). Comparison of traditional face-to-face teaching with synchronous e-learning in otolaryngology emergencies teaching to medical undergraduates: a randomised controlled trial. European Archives of Oto-RhinoLaryngology, 272(3), 759-763. doi:10.1007/s00405-014-3326-6

Alqahtani, M., \& Mohammad, H. (2015). Mobile applications' impact on student performance and satisfaction. Turkish Online Journal of Educational Technology-TOJET, 14(4), 102-112.

Bekele, T. A. (2010). Motivation and satisfaction in internet-supported learning environments: A review. Journal of Educational Technology \& Society, 13(2), 116-127. Obtenido de http://www.jstor.org/stable/jeductechsoci.13.2.116.

Bernal, C. (2010). Metodología de la investigación: administración, economía y ciencias sociales (Tercera ed.). Bogotá, Colombia: Pearson.

Chopra, G., Madan, P., Jaisingh, P., \& Bhaskar, P. (2019). Effectiveness of e-learning portal from students' perspective. Interactive Technology and Smart Education, 94-116. doi:10.1108/ITSE-05-2018-0027

Ebner, C., \& Gegenfurtner, A. (2019). Learning and satisfaction in webinar, online, and face-to-face instruction: a meta-analysis. Frontiers in Education, 1-11. doi:10.3389/feduc.2019.00092

Gegenfurtner, A., Zitt, A., \& Ebner, C. (2020). Evaluating webinar-based training: a mixed methods study of trainee reactions toward digital web conferencing. International Journal of Training and Development, 24(1), 5-21. doi:10.1111/ijtd.12167

Gschwandtner, M. (2016). The use of webinars for information skills training: evaluation of a one year project at Canterbury Christ Church University. Sconul Focus, 66, 56-61.

Hernandez Sampieri, R., Fernandez, C., \& Baptista, P. (2010). Metodologia de la investigacion (Sexta ed.). Mexico: MCGRAW HILL.

Khajuria, A. (2019). Evaluating the usefulness and utility of a webinar as a platform to educate students on a UK clinical academic programme. JR Coll Physicians Edinb, 49, 317-22. doi:10.4997/JRCPE.2019.415

Knipfer, C., Wagner, F., Knipfer, K., Millesi, G., Acero, J., Hueto, J., \& Nkenke, E. (2019). Learners' acceptance of a webinar for continuing medical education. International journal of oral and maxillofacial surgery, 48(6), 841-846. doi:https://doi.org/10.1016/j.ijom.2018.11.010

Kukreja, V., Kaur, A., \& Aggarwal, A. (2021). What factors impact Online Education? A factor analysis approach. Journal of Engineering Education Transformations, 34, 365-374. doi:10.16920/jeet/2021/v34i0/157180

Lee, J. (2014). An exploratory study of effective online learning: Assessing satisfaction levels of graduate students of mathematics education associated with human and design factors of an online course. International Review of Research in Open and Distributed Learning, 15(1), 111-132. doi:10.19173/irrodl.v15i1.1638

Mason, R., \& Weller, M. (2000). Factors affecting students' satisfaction on a web course. Australasian Journal of Educational Technology, 16(2), 173-200. doi:10.14742/ajet.1830

Munteanu, C., Ceobanu, C., Bobâlcă, C., \& Anton, O. (2010). An analysis of customer satisfaction in a higher education context. International Journal of Public Sector Management, 23(2), 124-140. doi:10.1108/09513551011022483/full/html

Oliver, E. (30 de Junio de 2020). Forbes México . Obtenido de https://www.forbes.com.mx/4mejores-software-para-videoconferencias-2020/

Pedró, F. (3 de Diciembre de 2020). Instituto Internacional de Educación Superior en América Latina $y$ el Caribe. Obtenido de IESALC UNESCO: https://www.iesalc.unesco.org/2020/12/03/reimaginar-la-universidad-en-la-pandemia/

Roff, K. A. (2018). Student Satisfaction and/or Dissatisfaction in Blended Learning Environments. Frontiers in Education Technology, 1(2), 149. doi:10.22158/fet.v1n2p149

Rositas, J. (2014). Los tamaños de las muestras en encuestas de las ciencias sociales y su repercusión en la generación del conocimiento. Innovaciones de negocioas , 11(22), 235-268. 
Senecal, J., \& Gazda, R. (2010). Harmonizing the Virtual Choir: Interactive Synchronous Webinars for Online Education. Journal of Interactive Instruction Development, 21(3), 13-16.

Topor, D. R., \& Budson, A. E. (2020). Twelve tips to present an effective webinar. Medical teacher, 42(11), 1216-1220. doi:10.1080/0142159X.2020.1775185

Zoumenou, V., Sigman-Grant, M., Coleman, G., Malekian, F., Zee, J. M., Fountain, B. J., \& Marsh, A. (2015). Identifying Best Practices for an Interactive Webinar. Journal of Family \& Consumer Sciences, 107(2), 62-69. Obtenido de https://www.ingentaconnect.com/content/aafcs/jfcs/2015/00000107/00000002/art00012 